\title{
ARCHIVIST
}

\section{Breastfeeding: sensitive mothers and intelligent offspring}

Could we persuade more mothers to breastfeed if we told them that their children would not only be more intelligent, but also earn more money? A well-publicised study from the city of Pelotas, Brazil, suggests that this is true (Victora CG et al. Lancet Glob Health 2015;3:e199205). They recruited over 5900 babies born in 1982 and were able to study nearly 3500 of them at the age of 30 years. They already had demographic data on their mothers, and at age 30 they tested their IQ and gathered data on duration of education and monthly income. Brazil was a particularly interesting setting for this research since in 1982, unlike many other countries, breastfeeding was equally prevalent across its widely divergent socioeconomic strata, and indeed, most women would have been unaware of its health benefits; thus there would have been little of the confounding by social class seen more developed countries. They found highly significant increases in all three measures associated with duration of breastfeeding, irrespective of whether it was exclusive. Compared to those who had breastfed for less than 1 month, those breastfed for 12 months or more had significantly higher IQ scores (mean 3.76 points; $95 \%$ CI 2.20-5.3), spent a mean 0.9 more years in education (95\% CI 0.42-1.40), and had higher mean monthly incomes of 341 Brazilian reals (approx. US\$105, UK $£ 70, € 95$ ): equivalent to about a third of the average income for that community. They did multiple regression analysis to allow for confounding variables, including family socioeconomic status at birth, maternal smoking, ethnicity and perinatal factors; the associations remained significant. The links between breastfeeding and IQ, and between education and income have been shown before, but never between breastfeeding and income directly, and never so long into adulthood. The long-term public health benefits of promoting breastfeeding are obvious.

But what is the mechanism? There is evidence that the nutrient content of breastmilk is of itself beneficial to brain development, but could the psychological effects be equally important? Co-incidentally, a study from Canada has revealed evidence that breastfeeding for more than 3 months is associated with increased maternal sensitivity, which in turn is associated with a more benign infant temperament at age 18 months (Jonas W et al. Acta Paed 2015. doi:10.1111/apa.12987). They recruited 170 mothers, and measured Maternal Sensitivity using a validated tool by video-recording interactions with their baby, and later Infant Temperament using the Early Childhood Behavioural Questionnaire, completed by the mothers. They allowed for the usual confounding factors, and included total duration and general attitude to breastfeeding: the association remained. Interestingly, the benefit of breastfeeding was greatest in those mothers with the highest anxiety scores. If calmer babies become higher-achieving adults, this might partially explain the Brazilian study's outcomes.

Competing interests None.

Provenance and peer review Commissioned; internally peer reviewed.

Accepted 9 April 2015

Published Online First 28 April 2015

Arch Dis Child 2015;100:601. doi:10.1136/archdischild-2015-308764 